\title{
Elasticity of the Coracoacromial Ligament in Shoulders with Rotator Cuff Tears: Measurement with Ultrasound Elastography
}

\author{
Hiroaki Kijima $^{1 *}$, Hiroshi Minagawa ${ }^{2}$, Tatsuru Tomioka ${ }^{3}$, Shin $\operatorname{Yamada}^{1}, \operatorname{Koji~Nozaka}^{1}$, \\ Hidetomo Saito ${ }^{1}$, Yoichi Shimada ${ }^{1}$ \\ ${ }^{1}$ Department of Orthopedic Surgery, Graduate School of Medicine, Akita University, Akita, Japan \\ ${ }^{2}$ Department of Orthopedic Surgery, Johto Orthopedic Clinic, Akita, Japan \\ ${ }^{3}$ Department of Orthopedic Surgery, Yokote Municipal Hospital, Yokote, Japan \\ Email: "h-kijima@gd5.so-net.ne.jp
}

Received June 16, 2013; revised July 18, 2013; accepted July 26, 2013

Copyright (C) 2013 Hiroaki Kijima et al. This is an open access article distributed under the Creative Commons Attribution License, which permits unrestricted use, distribution, and reproduction in any medium, provided the original work is properly cited.

\begin{abstract}
Background: The symptoms of rotator cuff tear vary and may even be absent. Thus, the symptoms cannot be explained solely by the presence of rotator cuff tear, and other factors are thought to be involved. Because the rotator cuff comes in contact with the coracoacromial ligament, the elasticity of the ligament may be a factor of the symptoms. The purpose of this study was to clarify the elasticity of the coracoacromial ligament in live shoulders with rotator cuff tears. Methods: Forty-one shoulders in 24 persons were enrolled in this study. The average age was 52 years old (range, 15 84 years old). We investigated the presence of rotator cuff tear and measured the elasticity of the coracoacromial ligament by ultrasound elastography (EUB-7500, HITACHI, Japan). Ultrasound elastography was a useful method to quantify the strain of soft tissue when pressure was added. The strain ratio (the ratio of strain of the coracoacromial ligament to that of the rotator cuff) was used as the index of the elasticity of coracoacromial ligament. Thus, the higher the strain ratio was, the softer the ligament was. Results: The strain ratio of the coracoacromial ligaments without rotator cuff tear showed a negative correlation to the age $(\mathrm{r}=-0.825, \mathrm{P}<0.01)$. The strain ratio of the ligaments with rotator cuff tear $(23.75 \pm 15.05,69.6$ years old $)$ was higher than that of the older ligaments without cuff tear $(12.62 \pm 7.94,64.6$ years old) $(\mathrm{P}=0.0486)$. In shoulders with rotator cuff tear, the strain ratio of the ligaments with pain $(14.37 \pm 10.15,66.7$ years old) was lower than that of ligaments without pain $(33.12 \pm 13.59,69.1$ years old $)(P=0.0221)$. Conclusions: The coracoacromial ligament became stiffened with aging but softened with the existence of rotator cuff tear. The ligaments with symptomatic cuff tear are more stiffened than the ligaments with asymptomatic cuff tear.
\end{abstract}

Keywords: Rotator Cuff Tear; Coracoacromial Ligament; Pain; Ultrasound Elastography

\section{Introduction}

Epidemiological studies have shown that low back pain, knee pain, and shoulder pain are common. The prevalence of low back pain and knee pain increases with aging, whereas shoulder pain tends to decrease at the peak in 50 - 60 generations [1-3].

Rotator cuff tear is a common cause of shoulder pain. The prevalence of rotator cuff tear also increases with aging [4]. However, symptoms of rotator cuff tear vary and may even be absent. Thus, the symptoms cannot be explained solely by the presence of rotator cuff tear, and other factors are thought to be involved. If these factors

"Corresponding author. are clarified, various approaches to pain reduction are possible.

Previous study of coracoacromial ligament elasticity with scanning acoustic microscopy, which can be used to evaluate the elasticity of tissue at the microscopic level, have shown that coracoacromial ligaments that show advanced degeneration have a higher elastic modulus [5]. This result suggests that because the coracoacromial ligament degenerates histologically and stiffens with aging, the contact pressure of the rotator cuff and the coracoacromial ligament increases and the prevalence of shoulder pain or rotator cuff tear increases with aging. Same previous study has also shown that the ligaments in shoulders with rotator cuff tears have a lower elastic modulus 
[5]. From this result, we can hypothesize that the coracoacromial ligament softens after the onset of rotator cuff tear, and that the prevalence of shoulder pain decreases in turn because the contact pressure of the ligaments and the rotator cuff decreases. To confirm these possibilities, it is necessary to examine the relationship between shoulder pain and the elasticity of the coracoacromial ligament in vivo. Many researchers have used ultrasound elastography to evaluate the elasticity of lesions of the breast, prostate, or liver in vivo [6-10]. The purpose of the present study was to clarify elastic changes with aging of the coracoacromial ligament and to clarify the relationship between the elasticity of the coracoacromial ligament and symptoms of rotator cuff tears by means of ultrasound elastography.

\section{Materials and Methods}

Forty-one shoulders (29 in male subjects and 12 in female subjects) in 24 healthy volunteers and patients, who had come to our hospital, were examined in this study. The average age of the subjects was 52 years (range, 15 84 years). Consent for this study was obtained from our institution, and informed consent was obtained from all subjects.

We first asked subjects whether they had shoulder pain and examined them whether their rotator cuff tore with an ultrasound scanner (EUB-7500, Hitachi, Tokyo, Japan). Next, the elasticity of coracoacromial ligament was assessed.

The shoulders were classified into 2 groups: an RCT group (12 shoulders) with rotator cuff tears, and a normal group (29 shoulders) without rotator cuff tears. Of the shoulders in the RCT group, those with pain were placed in the symptomatic group (6 shoulders), and those without pain were placed in the asymptomatic group ( 6 shoulders). Of the shoulders in the normal group, those of persons younger than 50 years old were placed in the younger group (19 shoulders), and those of persons older than 50 years old were placed in the older group (10 shoulders). All shoulders without rotator cuff tears had no symptoms. No significant difference was observed between the age of the older group and that of the RCT group or between the age of the symptomatic group and that of the asymptomatic group.

We classified into RCT group when the rotator cuff surface was concave or when the rotator cuff was not detected in longitudinal image of cuff tendon with ultrasonography. The predictive positive value of this method was reported previously to be $100 \%$ [11]. We did not measure the size of rotator cuff tear.

An ultrasound scanner (EUB-7500, Hitachi, Tokyo, Japan) was used to evaluate the elasticity of the coracoacromial ligament. Ultrasound elastography is a me- thod of assessing tissue elasticity on the basis of the relative strain caused when pressure is applied from the skin with the ultrasound probe. The strain ratio, which is the ratio of the strain of the coracoacromial ligament to the strain of the rotator cuff, was used as an index of coracoacromial ligament elasticity in this study. Therefore, the higher the strain ratio is, the softer the coracoacromial ligament is, because the coracoacromial ligament strains more easily than does the rotator cuff when the same amount of pressure is applied.

The longitudinal image of the coracoacromial ligament was depicted with the coracoid process and acromion as landmarks (Figure 1) and next, by turning the probe 90 degrees, the axial image of the ligament was depicted. When the position of the coracoacromial ligament on the axial image was inarticulate, this was confirmed by referring to the position of the thoracoacromial artery with Doppler imaging. The B-mode axial image and the elastographic image of the coracoacromial ligament was displayed on the right and left screens, respectively, of a 2-screen display (Figure 2). Then, we determined the strain ratio $\mathrm{B} / \mathrm{A}$, which was the ratio of the strain of the coracoacromial ligament (B) to the strain of the rotator cuff (A) (Figure 2). Pearson's correlation coefficients were calculated between ages and strain ratios, and Student's $t$-test was used to compare strain ratios within each group. Significance was set at the $\mathrm{P}<0.05$ level.

\section{Results}

The strain ratio of the normal group was negatively correlated with the subject's age $(\mathrm{r}=-0.825, \mathrm{P}<0.01$; Figure 3). The strain ratio of the RCT group was higher than that of the older group without a significant difference in the age of the RCT group (Figure 4). In the RCT group, the strain ratio of the symptomatic group was lower than that of the asymptomatic group (Figure 5).

\section{Discussion}

The coracoacromial ligament stiffened with aging. Therefore, this stiffened ligament may be a causative factor for rotator cuff tears because the ligament comes in contact with the rotator cuff. On the other hand, the coracoacromial ligament softened when a rotator cuff tear was present. This softening of the ligament might be related to spontaneous pain relief often observed in the natural history of rotator cuff tears, because ligaments in cases of asymptomatic cuff tear are softer than ligaments in cases of symptomatic cuff tear.

Ultrasound elastography, which was developed in 1991, can noninvasively quantify the strain of objects [12]. Ultrasound elastography detects displacements of organization when static pressure is applied. In other 


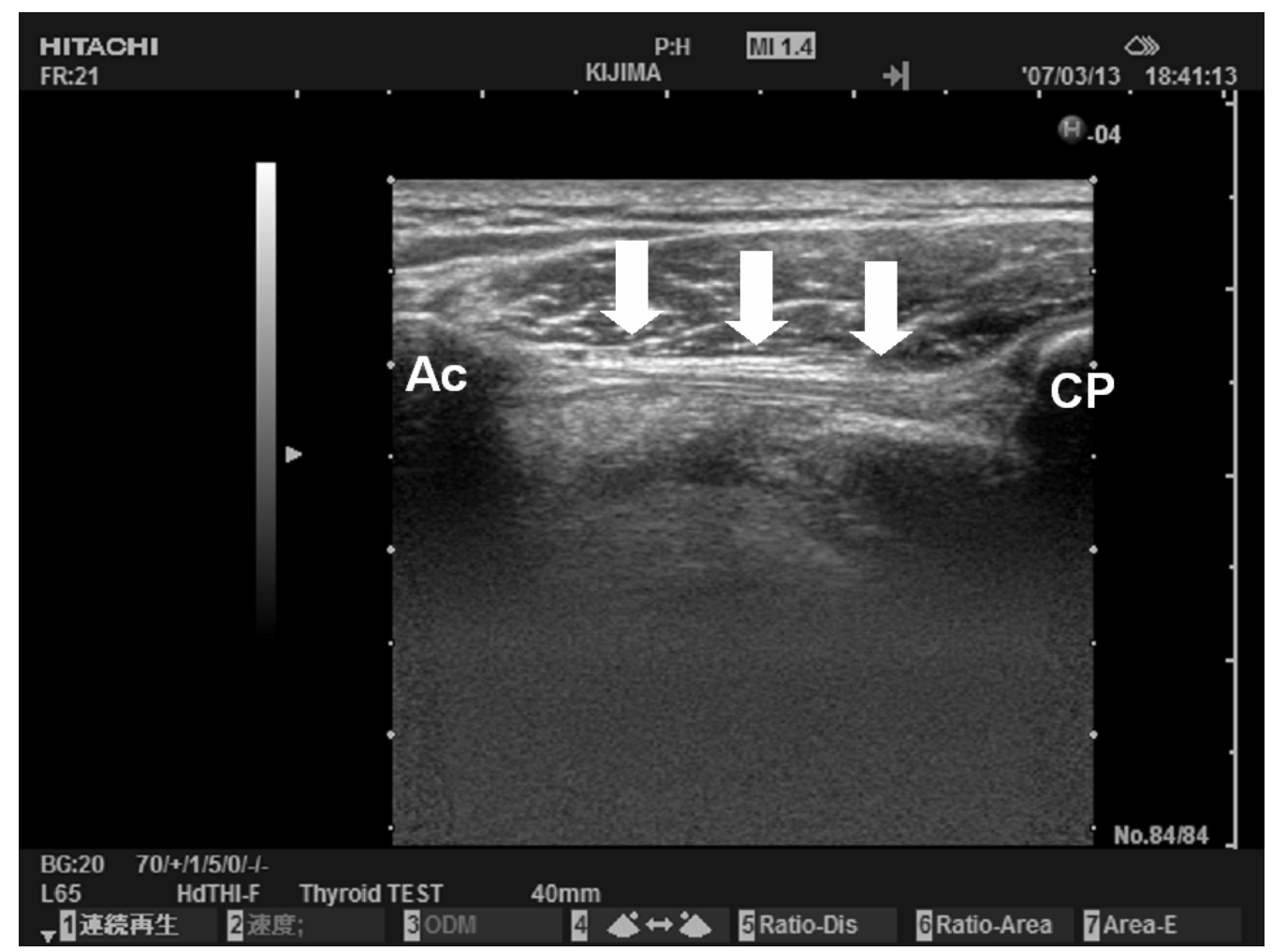

Figure 1. Ultrasonographic image of the coracoacromial ligament (B-mode longitudinal image). Ac: Acromion; CP: coracoid process; arrow: coracoacromial ligament.

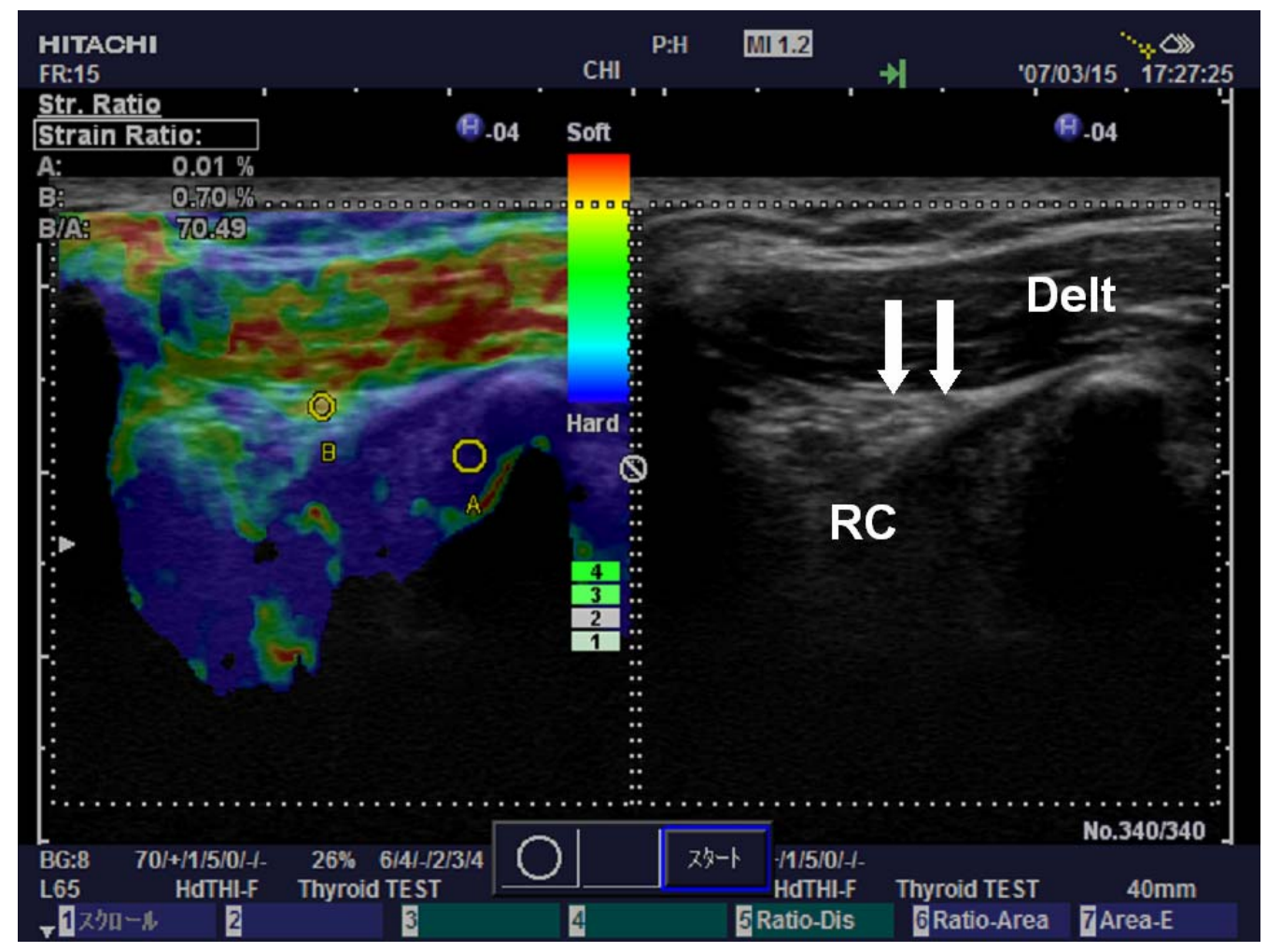

Figure 2. Ultrasound elastographic image. The right image is a B-mode axial image of the coracoacromial ligament, and the left image is an elastographic image of this ligament. RC: rotator cuff; Delt: deltoid muscle; and arrow: coracoacromial ligament. 


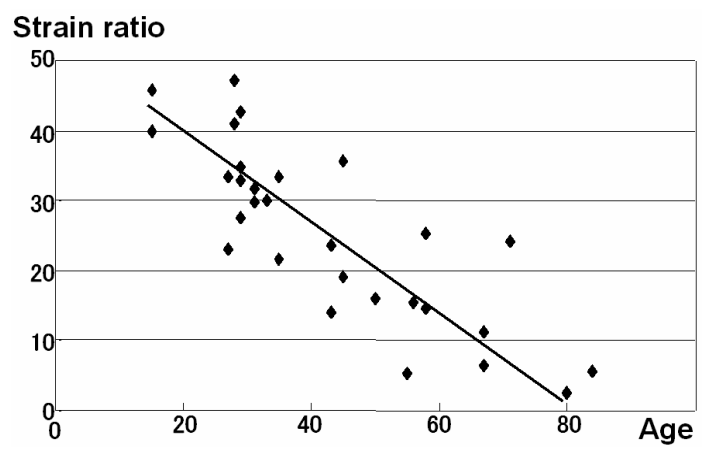

Figure 3. The relationship between the strain ratio and age in the normal group. The strain ratio of the normal group was negatively correlated with age $(r=-0.825, P<0.01)$.

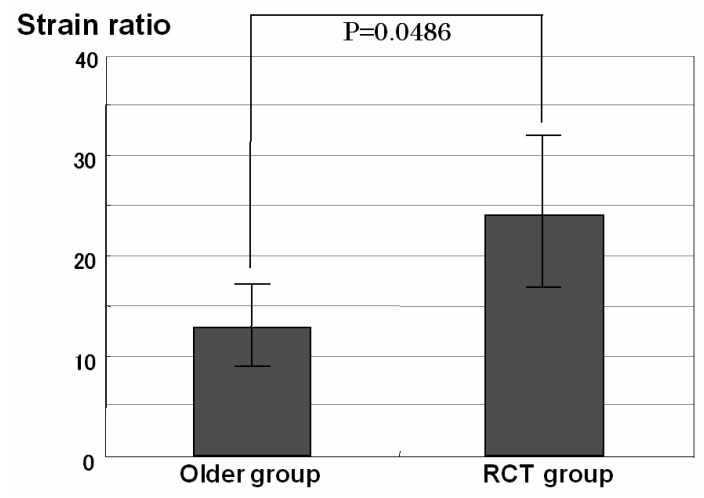

Figure 4. The difference in the strain ratio between shoulders with and without rotator cuff tears. The strain ratio of the RCT group was higher than that of the older group without a significant difference in the age of the RCT group.

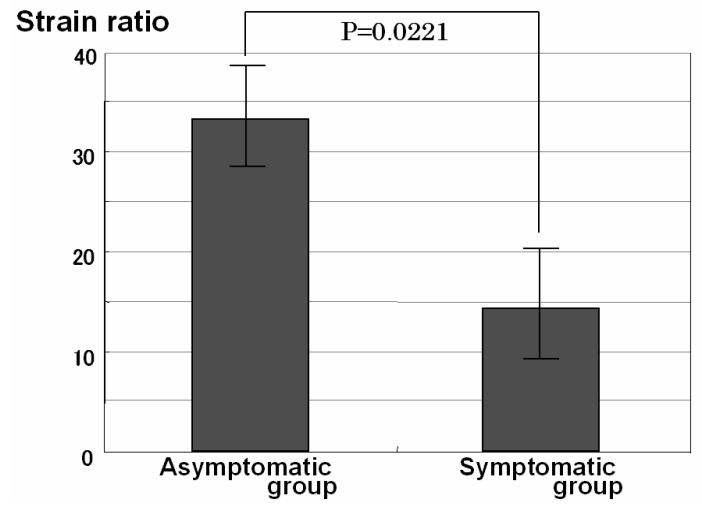

Figure 5. The difference in the strain ratio in shoulders with rotator cuff tears between those with and without pain. In the RCT group, the strain ratio of the symptomatic group was lower than that of the asymptomatic group.

words, the degree of tissue extension under external pressure is recorded with a high-resolution ultrasound scanner, and the elasticity of the tissue is evaluated through this correlation. Because ultrasound elastography can detect histological changes related to tissue elasticity in real time $[13,14]$, its effectiveness in the diagnosis of lesions of the prostate [6,7], breast [8,9], and liver [10] has been reported. To our knowledge, the present study is the first to use ultrasound elastography in orthopedics. This method is a useful technique that can be applied to many objects, such as muscles, tendons, and peripheral nerves, because it can distinguish organizations and quantify elasticity differently from the muscle rigidity meter, which is conventionally used to measure body hardness.

The strain ratio of the coracoacromial ligament was negatively correlated with age. This result indicates that the coracoacromial ligament stiffens with aging. Stiffening of the coracoacromial ligament causes an increase in the contact pressure between the coracoacromial ligament and the rotator cuff, and this increase in pressure may cause degeneration of the rotator cuff. In addition, stiffening of this ligament may lead to subacromial spur formation, because the subacromial spur has been recognized as a traction osteophyte of the coracoacromial ligament. Therefore, the stiffening of the coracoacromial ligament may be an etiological factor in rotator cuff tears and may support the finding that the prevalence of rotator cuff tears increases with aging.

The coracoacromial ligament is suggested to soften after a rotator cuff tore, because ligaments of shoulders with rotator cuff tears are softer than ligaments of agematched shoulders with a normal cuff. Previous histological study also suggested that the coracoacromial ligament was softened when its fiber structure collapsed as degeneration progressed with the rotator cuff tear [5]. And the present study supports that previous report, in vivo. In addition, the coracoacromial ligaments of shoulders with asymptomatic rotator cuff tears are particularly soft. Therefore, the softening of the coracoacromial ligament may be related to the development of asymptomatic rotator cuff tears. The subacromial bursa between the coracoacromial arch and the rotator cuff contains many free nerve endings. Softening of the coracoacromial ligament may decrease the contact pressure between the coracoacromial ligament and the rotator cuff. For rotator cuff tears, repair of the cuff is one treatment method. However, we can expect an effective treatment to decrease pressure under the coracoacromial ligament.

One limitation of the present study is that we have not considered other factors for example subacromial bursa, biceps tendon, or acromioclavicular joint. Another limitation of the present study is that the strain quantified with ultrasound elastography is a relative value. In this study, the strain ratio was useful for an index of the contact pressure between coracoacromial ligament and rotator cuff, because the strain ratio is not the hardness itself of coracoacromial ligament but the ratio of the easiness of strain of coracoacromial ligament to the easiness of strain of rotator cuff. And, the factor that we paid atten- 
tion to as one of the causes of the shoulder pain was a rise of the contact pressure between coracoacromial ligament and rotator cuff.

To apply the results of this study clinically, a technical development that will allow the elasticity to be evaluated as an absolute value in vivo is needed. If this technology is achieved, we conduct a same investigation in more numerous numbers, easily. When we can measure the elasticity of coracoacromial ligaments in vivo, the risk of rotator cuff tear or the possibility of pain relief can be predicted.

\section{REFERENCES}

[1] H. I. Andersson, G. Ejlertsson, I. Leden and C. Rosenberg, "Chronic Pain in a Geographically Defined General Population: Studies of Differences in Age, Gender, Social Class, and Pain Localization," The Clinical Journal of Pain, Vol. 9, No. 3, 1993, pp. 174-182. doi:10.1097/00002508-199309000-00004

[2] E. M. Badley and A. Tennant, "Changing Profile of Joint Disorders with Age: Findings from a Postal Survey of the Population of Calderdale, West Yorkshire, United Kingdom," Annals of the Rheumatic Diseases, Vol. 51, No. 3, 1992, pp. 366-371. doi:10.1136/ard.51.3.366

[3] M. Urwin, D. Symmons, T. Allison, T. Brammah, H. Busby, M. Roxby, A. Simmons and G. Williams, "Estimating the Burden of Musculoskeletal Disorders in the Community: The Comparative Prevalence of Symptoms at Different Anatomical Sites, and the Relation to Social Problem," Annals of the Rheumatic Diseases, Vol. 57, No. 11, 1998, pp. 649-655. doi:10.1136/ard.57.11.649

[4] A. Yamamoto, K. Takagishi, T. Osawa, T. Yanagawa, D. Nakajima, H. Shitara and T. Kobayashi, "Prevalence and Risk Factors of a Rotator Cuff Tear in the General Population," Journal of Shoulder and Elbow Surgery, Vol. 19, No. 1, 2010, pp. 116-120. doi:10.1136/ard.57.11.649

[5] H. Kijima, H. Minagawa, Y. Saijo, H. Sano, T. Tomioka, N. Yamamoto, Y. Shimada, K. Okada and E. Itoi, "Degenerated Coracoacromial Ligament in Shoulders with Rotator Cuff Tears Shows Higher Elastic Modulus: Measurement Using Scanning Acoustic Microscopy," Journal of Orthopaedic Science, Vol. 14, No. 1, 2009, pp. 62-67. doi:10.1007/s00776-008-1283-x

[6] A. Lorenz, H. Ermert, H. J. Sommerfeld, M. Garcia-
Schürmann, T. Senge and S. Philippou, "Ultrasound Elastography of the Prostate: A New Technique for Tumor Detection," Ultraschall in der Medizin, Vol. 21, No. 1, 2000, pp. 8-15. doi:10.1055/s-2000-8926

[7] D. Cochlin, R. H. Ganatra and D. F. R. Griffiths, "Elastography in the Detection of Prostatic Cancer," Clinical Radiology, Vol. 57, No. 11, 2002, pp. 1014-1020. doi:10.1053/crad.2002.0989

[8] J. Bercoff, S. Chaffai, M. Tanter, L. Sandrin, S. Catheline, M. Fink, J. L. Gennisson and M. Meunier, "In Vivo Breast Tumor Detection Using Transient Elastography," Ultrasound in Medicine \& Biology, Vol. 29, No. 10, 2003, pp. 1387-1396. doi:10.1016/S0301-5629(03)00978-5

[9] A. Thomas, T. Fischer, H. Frey, R. Ohlinger, S. Grunwald, J. U. Blohmer, K. J. Winzer, S. Weber, G. Kristiansen, B. Ebert and S. Kümmel, "Real-Time Elastography; an Advanced Method of Ultrasound: First Results in $108 \mathrm{~Pa}-$ tients with Breast Lesions," Ultrasound in Obstetrics \& Gynecology, Vol. 28, No. 3, 2006, pp. 335-340. doi:10.1002/uog.2823

[10] T. Varghese and H. Shi, "Elastographic Imaging of Thermal Lesions in Liver In-Vivo Using Diaphragmatic Stimuli," Ultrasonic Imaging, Vol. 26, No. 1, 2004, pp. 18-28. doi:10.1177/016173460402600102

[11] T. Tomioka, H. Minagawa, N. Yamamoto, H. Abe, K. Kikuchi, H. Kijima and E. Itoi, "Diagnostic Value of Portable Ultrasonography in Detecting Full-Thickness Rotator Cuff Tears," The Shoulder Joint, Vol. 31, 2006, p. 146.

[12] W. Khaled, S. Reichling, O. T. Bruhns and H. Ermert, "Ultrasonic Strain Imaging and Reconstructive Elastography for Biological Tissue," Ultrasonics, Vol. 44, Supplement 1, 2006, pp. e199-e202. doi:10.1016/j.ultras.2006.06.007

[13] E. Konofagou and J. Ophir, "A New Elastographic Method for Estimation and Imaging of Lateral Displacements, Lateral Strains, Corrected Axial Strains and Poisson's Ratios in Tissues," Ultrasound in Medicine \& Biology, Vol. 24, No. 8, 1998, pp. 1183-1199. doi:10.1016/j.ultras.2006.06.007

[14] T. A. Krouskop, T. M. Wheeler, F. Kallel, B. S. Garra and T. Hall, "Elastic Moduli of Breast and Prostate Tissues under Compression," Ultrasonic Imaging, Vol. 20, No. 4, 1998, pp. 260-274. doi:10.1016/j.ultras.2006.06.007 